\title{
Simultaneous observations of the saturnian aurora and polar haze with the HST/FOC
}

\author{
J.C. Gérard, V. Dols and D. Grodent \\ LPAP, Institut d'Astrophysique, Université de Liège, Belgium
}

J.H. Waite and G.R. Gladstone

Southwest Research Institute, San Antonio, Texas

\section{R. Prangé}

Institut d'Astrophysique Spatiale, CNRS, Orsay and Institut d'Astrophysique de Paris, France

\begin{abstract}
Near simultaneous observations of the Saturnian $\mathrm{H}_{2}$ north ultraviolet aurora and the polar haze were made at $153 \mathrm{~nm}$ and $210 \mathrm{~nm}$ respectively with the Faint Object Camera on board the post-COSTAR Hubble Space Telescope. The auroral observations cover a complete rotation of the planet and, when co-added, they reveal the presence of an auroral emission near $80^{\circ} \mathrm{N}$ with a brightness of about $150 \mathrm{kR}$ of total $\mathrm{H}_{2}$ emission. The maximum vertical optical depth at 210 $\mathrm{nm}$ is found to be located $\sim 5^{\circ}$ equatorward of the auroral emission zone. The haze particles are presumably formed by hydrocarbon aerosols initiated by $\mathrm{H}_{2}^{+}$auroral production. In this case, the $3 \times 10^{10} \mathrm{~W}$ of $\mathrm{H}_{2}$ emission observed with the FOC, combined with the deduced haze optical depth requires an efficiency of aerosol formation of about $7 \%$. This result indicates that auroral production of hydrocarbon aerosols is a viable source of high-latitude haze.
\end{abstract}

\section{Introduction}

The ultraviolet aurora of Saturn has been studied much less extensively than the Jovian aurora. Spatiallyresolved spectra were obtained with the International Ultraviolet Explorer (IUE) satellite (Clarke et al., 1980). The first unambiguous evidence for the Saturnian aurora was provided by the UV spectrometer (UVS) aboard the Voyager spacecraft (Broadfoot et al., 1981; Sandel and Broadfoot, 1981; Sandel et al., 1981). The UVS measured auroral emission of Ly- $\alpha$ and $\mathrm{H}_{2}$ Lyman and Werner bands from the polar regions of both hemispheres. Latitudinal slit scans across the south pole located the emission between $78^{\circ} \mathrm{S}$ and $81.5^{\circ} \mathrm{S}$ at Saturn System III longitude $=190^{\circ}$. Based on an assumption about the extent of the auroral oval, the nadir brightness in the oval was estimated $\simeq 2.8 \mathrm{kR}$. Both observations showed a maximum at $50^{\circ}<\lambda_{I I I}<180^{\circ}$. They

Copyright 1995 by the American Geophysical Union.

Paper number 95GL02645

0094-8534/95/95GL-02645\$03.00 were also consistent with a brightening when the longitude of the mean meridian is near $50^{\circ}$ to $100^{\circ}$.

In parallel, observations made at $265 \mathrm{~nm}$ in the polar regions of giant planets with the photopolarimeter (PPS) aboard Voyager 2 were analyzed by West et al. (1983) and Pryor and Hord (1991). The darkening of the polar region is ascribed to the presence of stratospheric haze. The observed northern haze region appears to enclose the auroral zone as defined from the UVS morphological observations. Recently, Ben Jaffel et al. (1995), using FOC-HST pre-COSTAR images, showed a good correlation between the peak of the haze absorption at $210 \mathrm{~nm}$ and the UVS location of the aurora.

\section{Observations}

The observations were made between 22:53 UT October 1 and 11:42 UT October 2, 1994 with the FOC $\mathrm{F} / 96$ relay. The $1024 \times 512 \mathrm{Z}$ zoomed pixel format was used to observe with the largest possible field of view $(14 \times 14 \mathrm{arcsec})$. A first $1195 \mathrm{~s}$ exposure was made with the F210M + F220W filter setup which isolates a 20 $\mathrm{nm}$ wide region centered at $210 \mathrm{~nm}$. To avoid excessive count rate leading to non-linearity of the detector's response, the neutral filter F1ND was also used in series with the UV filters. The purpose of this image was both to get a good determination of the UV planetary limb and to analyze the dark haze distribution in the polar cap. A series of eight (seven $1075 \mathrm{~s}$ and one $895 \mathrm{~s}$ exposures, each separated by one HST orbit (96 $\mathrm{min}$ ) were subsequently made with the F152M + F175W filters in an attempt to image the UV Saturnian aurora over a complete planetary rotation. This filter passband isolates a $20 \mathrm{~nm}$ region centered at $153 \mathrm{~nm}$ which includes lines and continuum from the B ${ }^{1} \Sigma_{u}-\mathrm{X}^{1} \Sigma_{g}^{+}$Lyman transition. This filter combination provides good contrast against the sunlight reflected by the planetary disk (Gérard et al., 1994 and references therein). The nominal pointing of the telescope was such that the center of the FOC field of view tracked a point located at a planetographic latitude of $40^{\circ} \mathrm{N}$ (37 $7^{\circ}$ planetocentric) on the central meridian. During the observations, the phase 
angle of Saturn was $3.1^{\circ}$ and the apparent equatorial and polar diameters were 18.8 and 16.7 arcsec respectively. The planet rotated by $10.5^{\circ}$ during each $1075 \mathrm{~s}$ exposure.

\section{Results}

\section{Auroral morphology}

No single image at $153 \mathrm{~nm}$ shows a clear auroral contribution. To improve its detectability, all 8 exposures at $153 \mathrm{~nm}$ were added together.

The characteristics of the auroral morphology are best illustrated in Figure 1 which shows an enlarged and contrast-enhanced version of the average of the $8 \mathrm{im}$ ages. We characterize the zones of auroral emission by the density and brightness of pixels defining them. Therefore, a test is applied to each pixel to check the count level and the number of neighboring pixels above a prescribed level and its intensity is increased to provide a better contrast. For comparison, this image overlays the $210 \mathrm{~nm}$ image which shows a well defined dark polar region extending down to about $65^{\circ} \mathrm{N}$. An alignment of bright features representing the most intense spots of the auroral emission closely follows the $80^{\circ}$ parallel and is interpreted as the brightest parts of the UV auroral arc previously detected with the Voyager UVS.

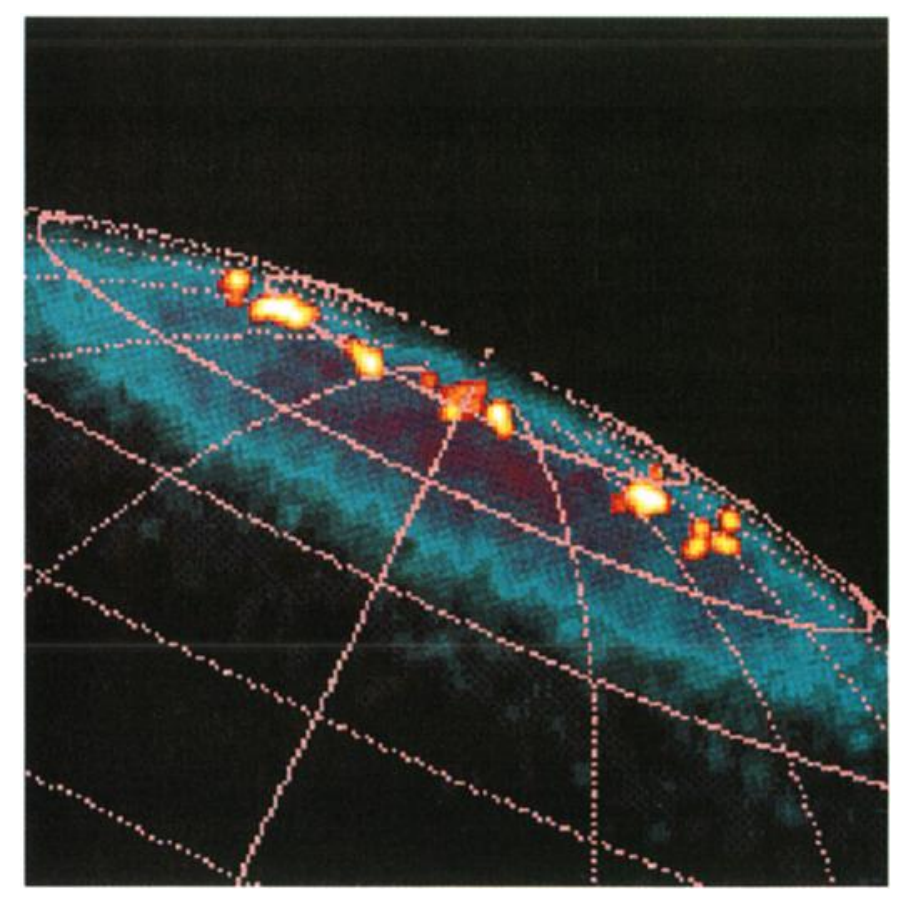

Figure 1 Comparison of the location of the auroral emission with the UV dark polar region. The opacity derived from the non-auroral image at $210 \mathrm{~nm}$ (bluepurple) is overlaid with the contrast-enhanced zones of emission at $153 \mathrm{~nm}$ (red-yellow). A grid of parallels and meridians is superimposed on the synthetic image. Parallels are separated by $10^{\circ}$ and meridians by $20^{\circ}$ of longitude.
Apparent auroral emission rates may be derived from the count rate in the emission zone using the FOC absolute calibration combined with the filter transmissions. The mean auroral count level above the disk background in the auroral region described before is on the order of $0.2 \mathrm{C} \mathrm{pix}^{-1}$ for the average duration of an exposure. The total instrument sensitivity in this configuration is $\sim 680 \mathrm{MR}$ (megarayleigh) $/ \mathrm{C} \mathrm{pix}^{-1} s^{-1}$ of total $\mathrm{H}_{2}$ emission (Lyman + Werner bands). This value is based on a detailed calculation of the FOC response to a synthetic unabsorbed spectrum of the $\mathrm{H}_{2}$ UV emission excited by $100 \mathrm{eV}$ electrons described in Trafton et al. (1994). Therefore, the average emission level is on the order of $150 \mathrm{kR}$. This value is larger than the peak values of 40-50 kR detected by the Voyager 1 and 2 UVS observations near $\lambda_{I I I}=150^{\circ}$. It should be stressed, however, that the UVS estimate was based on the assumption that the instrument slit was uniformly filled with auroral emission extending between $78^{\circ}$ and $81.5^{\circ}$. The FOC value reported here refers to a more localized region and could therefore be compatible with the UVS observations.

\section{Estimate of the total auroral power}

The total power radiated by the north aurora may be derived from the integrated count rate of the auroral emission observed at $153 \mathrm{~nm}$. A two-step procedure is applied : (1) the total auroral count rate above the disk background is derived from the data displayed in Figure 1 and (2) the count rate is converted into the total power radiated in the $\mathrm{H}_{2}$ bands and into a precipitated energy flux. Step 1 first requires a determination of the extent of the auroral emission zone. This determination is based on the contrast enhancement method outlined before. A mask of the emission zone is built and the total number of counts inside it is determined. Using the absolute camera sensitivity and a synthetic $\mathrm{H}_{2}$ spectrum, we estimate the total $\mathrm{H}_{2}$ (Werner and Lyman bands) full hemispheric radiated power to be $3 \times$ $10^{10} \mathrm{~W}$. If an efficiency factor of 7 (Gérard and Singh, 1982; Waite et al. 1983) is used for the conversion of the electron energy flux to the $\mathrm{H}_{2}$ emission, about $2 \times$ $10^{11} \mathrm{~W}$ must be deposited in the north polar region by the energetic electrons. A precipitated power of $\sim 2 \times$ $10^{11} \mathrm{~W}$ was derived from the Voyager 1 UVS observations (Broadfoot et al., 1981) for the two hemispheres. The fair agreement between the two sets of observations may be fortuitous, considering the rather poor statistics, possible sources of calibration errors and the high level of variability observed with the Voyager UVS.

\section{Correlation with the polar darkening}

As mentioned before, the UV darkening of the polar cap is generally explained by the presence of an additional stratospheric haze layer (West et al., 1983; Karkoschka and Tomasko, 1993) possibly generated by the interaction of the auroral energetic precipitation with the Saturnian atmosphere (Pryor and Hord, 1991). 

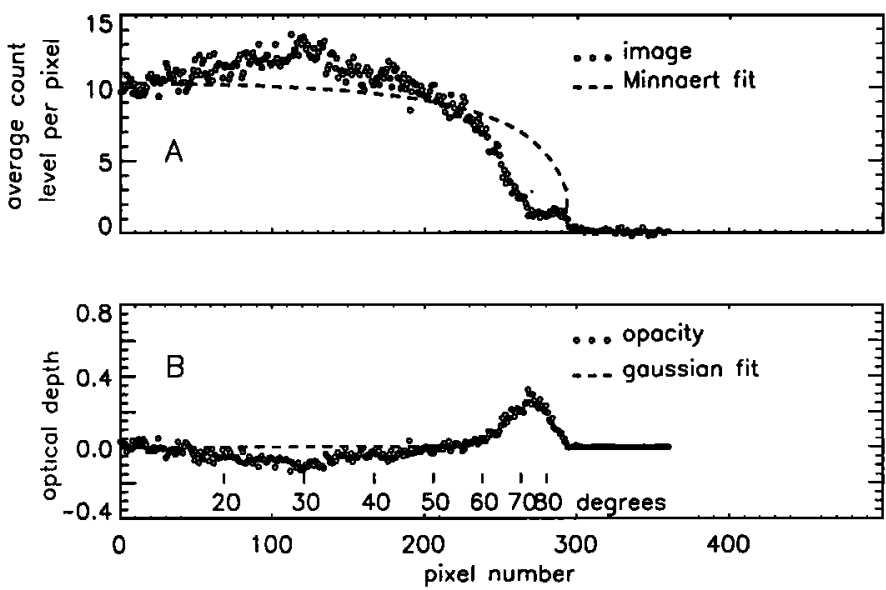

Figure 2 (a) Center-to-limb variation of Saturn's measured brightness at $210 \mathrm{~nm}$. The dots show the brightness variation measured in a 11-pixel wide cut along the central meridian in the raw image. The dashed line is the fit of a generalized Minnaert function to the data outside the dark polar cap region.(b) dots : distribution along the central meridian of the optical depth at 210 $\mathrm{nm}$ derived from (a) (see text). Dashed line : gaussian fit to the individual data points.

The present set of data provides a unique opportunity to compare the structures of the auroral UV emission and the UV darkening observed quasi simultaneously. The $210 \mathrm{~nm}$ image may be used to map the reflectivity I/F of the Saturnian atmosphere. To characterize the distribution of intensity in the dark north polar region, we consider a radial section from the Sub-Earth point along the central meridian to the north pole. It is shown in Figure 2a where the slight increase in brightness from the center to about $30^{\circ}$, probably the result of an inadequate flat field correction. To analyze the distribution of the absorption in the north polar region, we assume that the polar haze forms a purely absorbing layer located above the reflecting lower atmosphere. In this simple case, the additional absorption by the haze layer is given by $\left[\exp \left(-\frac{\tau}{\mu_{0}}\right) \exp \left(-\frac{\tau}{\mu}\right)\right]$, where $\tau$ is the polar haze vertical optical depth at $210 \mathrm{~nm}, \mu_{0}$ and $\mu$ the local cosines of the solar zenith angle and emission angle, respectively. The procedure adopted is thus to fit the reflectivity distribution of the disk, after removing the polar and ring regions, with a generalized Minnaert function (e.g. West et al., 1995). The synthetic Minnaert distribution $(\mathrm{I} / \mathrm{F})_{M \text { in }}$ is then divided by the observed reflectivity $(\mathrm{I} / \mathrm{F})_{o b s}$ along the central meridian. This ratio is close to unity except in the polar latitudes. The depth of the polar haze is given by:

$$
\tau=\ln \left(\frac{I_{M i n}}{I_{o b s}}\right)\left(\frac{1}{\mu_{0}}+\frac{1}{\mu}\right)^{-1}
$$

where $\mathrm{I}_{M i n}$ and $\mathrm{I}_{o b s}$ are respectively the Minnaert-fitted and the observed distribution directly derived from the $210 \mathrm{~nm}$ image. The latitudinal variation of the optical thickness along the central meridian derived from (1) is shown in figure $2 b$, together with a least square gaus- sian fit. The signal to noise ratio of data point in figure 2 (averaged over 22 pixels) varies from about 10 at midlatitudes to about 5 near the haze maximum absorption. Values of $\tau$ range from less than 0.1 at latitudes lower than $60^{\circ}$ to about 0.27 at $75^{\circ}$. A well defined maximum in $\tau$ is located at $75^{\circ}$, that is about $5^{\circ}$ equatorward of the auroral emission.

\section{Auroral production of aerosols}

Several sources of high-latitude aerosol production have been suggested by Pryor and Hord (1991) based on the interpretation of the Voyager Photopolarimeter Subsystem. The most plausible appears to be hydrocarbon aerosol formation initiated from aurorally produced $\mathrm{H}_{2}^{+}$ionization. This process is regulated by the production rate of $\mathrm{H}_{2}^{+}$ions efficiently converted into $\mathrm{H}_{3}^{+}$which react with $\mathrm{CH}_{4}$ to initiate polymerization. The relative altitude of the methane homopause and the region of maximum auroral electron energy deposition play an important role in determining the efficiency of this auroral aerosol formation process. The large quantities of atomic hydrogen formed from auroral impact of $\mathrm{H}_{2}$ will terminate any neutral polymerization schemes through three body recombination reactions of $\mathrm{CH}_{3}$ and $\mathrm{H}$ to reform $\mathrm{CH}_{4}$. However, ions such as $\mathrm{C}_{2} \mathrm{H}_{3}^{+}$can further react with $\mathrm{C}_{2} \mathrm{H}_{2}$ and continue toward polymerization. We estimate the efficiency of aerosol formation required to produce the observed haze as follows (cf. Pryor and Hord, 1991). Assuming that the haze is made up of spherical particles of radius $\mathrm{r}$ and density $\rho$, the column mass density of haze implied by our derived peak optical depth of $\tau \simeq 0.27$ is given by :

$$
N\left(\mathrm{~g} \mathrm{~cm}^{-2}\right) \sim 2 \times 10^{-6} \quad\left[\frac{\rho}{1}\left(\mathrm{~g} \mathrm{~cm}^{-3}\right)\right]\left[\frac{r}{0.1}(\mu \mathrm{m})\right]
$$

The lifetime of the haze is probably governed by the timescale for gravitational settling through the stratosphere (in the presence of eddy diffusion). A lifetime of $\sim 30$ years is estimated for such particles from West et al., (1995), so that maintenance of the aerosol layer would require a column mass production rate of

$$
P\left(g \mathrm{~cm}^{-2} \mathrm{~s}^{-1}\right) \sim 2 \times 10^{-15} \quad\left[\frac{\rho}{1}\left(g \mathrm{~cm}^{-3}\right)\right]\left[\frac{r}{0.1}(\mu \mathrm{m})\right]
$$

We assume that the aerosol haze is made of $0.1 \mu \mathrm{m} \mathrm{ra}-$ dius particles with a density $\simeq 1$ and extends from the pole to $64^{\circ} \mathrm{N}$, covering about $2.3 \times 10^{19} \mathrm{~cm}^{2}$. Thus, we derive an aerosol production rate integrated over the haze cap $\simeq 4.6 \times 10^{4} \mathrm{~g} \mathrm{~s}^{-1}$. The total production rate of $\mathrm{H}_{2}^{+}$ions in a $3 \times 10^{10} \mathrm{~W} \mathrm{H}_{2}$ aurora is about $3 \times$ $10^{28}$ ions $\mathrm{cm}^{-2}$ since the formation of each $\mathrm{H}_{2}^{+}$ions requires an average energy of $37 \mathrm{eV}$ (Waite et al. 1983). If we assume that (1) the aerosol composition is dominated by carbon and (2) that all ions producted below the methane homopause will result in the formation of hydrocarbon ions, we conclude that a typical Saturnian aurora is able to produce the observed haze layer if the aerosol formation (e.g. condensation or clustering on each hydrocarbon ion) is about $7 \%$. 
Based on an aerosol production-loss balance, Pryor and Hord (1991) derived, depending on the loss rate estimate, an efficiency ranging from 20 to $120 \%$ for a $1 \mathrm{kR}$ aurora extending over a $2 \times 10^{18} \mathrm{~cm}^{2}$ area and a polar haze zone north of $68^{\circ} \mathrm{N}$. We note that a 0.1 $\mu$ m-radius aerosol particle would contain about $2 \times 10^{8}$ carbon atoms, so that the process of going from hydrocarbon ions to aerosols is extremely poorly defined. If the single scattering albedo of the particles was non negligible, light scattered by the haze would only add a small amount to the efficient Rayleigh scattering by $\mathrm{H}_{2}$. In this case, our estimate of 0.27 for the maximum absorption opacity would be unchanged but the haze column estimate would need to be somewhat revised upward. Our results are merely to demonstrate that auroral production of hydrocarbon aerosols is a viable source for the high-latitude haze.

\section{Discussion}

The first images of the Saturnian UV aurora have been obtained with the post-COSTAR HST-FOC. Its local brightness maximum is larger than the values derived from the UVS spectrometer observations aboard Voyager but the total emitted power deduced from the two instruments are in fairly good agreement. The minimum reflectivity of the dark polar cap, presumably associated with the maximum optical depth $(\simeq 0.3)$ of the polar stratospheric haze, is found near $72^{\circ}-75^{\circ}$, that is equatorward of the UV auroral peak. Since these two sets of observations were made during a single Saturnian rotation and pointing was using the same guide stars, this conclusion is fairly robust. The $5^{\circ}-8^{\circ}$ shift found here between the presumed auroral haze source and the maximum haze absorption may possibly be ascribed to either (1) meridional transport of the precursors of the haze particles by equatorward meridional winds or (2) the possibility that the haze latitude represents the average latitude for the aurora, and we have observed a particular aurora at a higher latitude. Since a similar shift in latitude between the haze and the aurora was noticed by Pryor and Hord (1991), we favor the first possibility. Such winds would be easily generated by the large heat input into the auroral region from precipitating energetic particles. For example, a $100 \mathrm{kR} \mathrm{H}_{2}$ aurora corresponds to a particle energy flux of about 7 erg $\mathrm{cm}^{-2} \mathrm{~s}^{-1}$ about $50 \%$ of which is converted into gas heating. The latitudinal temperature gradient associated with the auroral heating rate excess over the solar EUV heating will redistribute heat by the combined effect of vertical and horizontal transport in a way similar to the Earth's and probably the Jovian aurora. Finally, as mentioned before, haze particles would only be efficiently produced by auroral particles if their energy is large enough to create sufficient ionization below the homopause. This assumption remains to be substantiated by observations.

Acknowledgments. The authors are grateful to L. Ben Jaffel for useful discussions and valuable suggestions about the data analysis. J.C.G. is supported by the Belgian Fund for Scientific Research. Funding for this research was provided by the PRODEX program of the Belgian Federal Office for Scientific, Technical and
Cultural Affairs, STScI grants AR-4916.01-92A, GO5341.01-93A and NASA Planetary Atmospheres NAGW 3624. These results are based on observations with the NASA/ESA Hubble Space Telescope, obtained at the STScI, which is operated by AURA, Inc., for NASA under contract NAS5-26555.

\section{References}

Ben Jafflel, L. , V. Leers, and B.R. Sandel, Dark auroral oval on Saturn discovered in Hubble Space Telescope ultraviolet images, Science, 269, 951, 1995.

Broadfoot, A.L. et al., Extreme ultraviolet observations from Voyager 1 encounter with Saturn, Science, 212, 206, 1981.

Clarke, J.T., H.W. Moos, S.K. Atreya and A.L. Lane, IUE detection of bursts of $\mathrm{H}$ Lyman-alpha from Saturn , $\mathrm{Na}$ ture , 290, 226, 1981.

Gérard, J.C., D. Grodent, R. Prangé, J.H. Waite, G.R. Gladstone, V. Dols, F. Paresce, A. Storrs, L. Ben Jaffel and K.A. Franke, A remarkable auroral event on Jupiter observed in ultraviolet with the Hubble Space Telescope, Science, 266, 1675, 1994.

Gérard, J.C. and Singh, V., A model of energetic electrons and EUV emission in the Jovian and Saturnian Atmospheres and implications, J. Geophys. Res., 87, 4525, 1982.

Karkoschka, E. and M.G. Tomasko, Saturn's upper atmospheric hazes observed by the Hubble Space Telescope, Icarus, 106, 428, 1993.

Lane, A.L. et al, Photopolarimetry for Voyager 2 : preliminary results on Saturn, Titan, and the rings, Science, 215, 537, 1982.

Pryor, W.R. and C.W. Hord, A study of Photopolarimeter system UV absorption data on Jupiter, Saturn, Uranus and Neptune : implications for auroral haze formation, Icarus, 91, 161, 1991.

Sandel, B.R. et al., Extreme ultraviolet observations from the Voyager 2 encounter with Saturn, Science, 215, 548, 1982.

Sandel, B.R. and A.L. Broadfoot, Morphology of Saturn's aurora, Nature, 292, 679, 1981.

Trafton, L.M., J.C. Gérard, G. Munhoven and J.H. Waite, High-resolution spectra of Jupiter's northern auroral ultraviolet emission with the Hubble Space Telescope, $A p$. $J ., 421,816,1994$.

Waite, J.H., T.E. Cravens, J. Kozyra, A.F. Nagy, S.K. Atreya and R.H. Chen, Electron precipitation and related aeronomy of the Jovian thermosphere and ionosphere, $J$. Geophys. Res., 88, 6143, 1983.

West, R.A., M. Sato, H. Hart, A.L. Lane, C.W. Hord, K.E. Simmons, L.W. Esposito, D.L. Coffeen and R.B. Pomphrey, Photometry and polarimetry of Saturn at 2640 and $7500 \AA, J$. Geophys. Res, 88, 8679, 1983.

West, R.A., E. Karkoschka, A.J. Friedson, M. Seymour, K. H. Baines and H.B. Hammel, Impact debris particles in Jupiter's stratosphere, Science, 267, 1296, 1995.

J.C. Gérard, V. Dols and D. Grodent, Laboratoire de Physique Atmosphérique et Planétaire, Institut d'Astrophysique, Université de Liège, 5, avenue de Cointe, B-4000 Liège, Belgium. (e-mail: gerard@astro.ulg.ac.be) J.H. Waite and G.R. Gladstone, Southwest Research Institute, San Antonio, Texas, 78228-0570, USA

R. Prangé, Institut d'Astrophysique Spatiale, CNRS, Université de Paris XI, Orsay and Institut d'Astrophysique de Paris, CNRS, France

(received March 2, 1995; revised May 24, 1995; accepted June 23, 1995.) 